\title{
ARTICLE OPEN Predicting the structure and stability of titanium oxide electrides
}

\author{
Xin Zhong $\mathbb{D}^{1,2,3}$, Meiling $\mathrm{Xu}^{4}$, Lili Yang ${ }^{1,2}$, Xin $\mathrm{Qu}^{1,2}$, Lihua Yang ${ }^{1,2}$, Miao Zhang ${ }^{5}$, Hanyu Liu (D) ${ }^{6}$ and Yanming Ma ${ }^{6,7}$
}

The search for new inorganic electrides has attracted significant attention due to their potential applications in transparent conductors, battery electrodes, electron emitters, as well as catalysts for chemical synthesis. However, only a few inorganic electrides have been successfully synthesized thus far, limiting the variety of electride examples. Here, we show the stabilization of inorganic electrides in the Ti-rich $\mathrm{Ti}-\mathrm{O}$ system through first-principles calculations in conjunction with swarm-intelligence-based CALYPSO method for structure prediction. Besides the known Ti-rich stoichiometries of $\mathrm{Ti}_{2} \mathrm{O}, \mathrm{Ti}_{3} \mathrm{O}$, and $\mathrm{Ti}_{6} \mathrm{O}$, two hitherto unknown $\mathrm{Ti}_{4} \mathrm{O}$ and $\mathrm{Ti}_{5} \mathrm{O}$ stoichiometries are predicted to be thermodynamically stable at certain pressure conditions. We found that these $\mathrm{Ti}-$ rich Ti-O compounds are primarily zero-dimensional electrides with excess electrons confined in the atom-sized lattice voids or between the cationic layers playing the role as anions. The underlying mechanism behind the stabilization of electrides has been rationalized in terms of the excess electrons provided by Ti atoms and their accommodation of excess electrons by multiple cavities and layered atomic packings. The present results provide a viable direction for searching for practical electrides in the technically important Ti-O system.

npj Computational Materials (2018)4:70; https://doi.org/10.1038/s41524-018-0131-6

\section{INTRODUCTION}

Electrides $^{1-3}$ are kind of peculiar ionic compounds, where the intrinsic excess electrons are loosely localized in the cavities or channels of the structure, behaving as anions in the cationic framework. The anionic electrons in the electrides are markedly different from the delocalized electrons in metals or entirely localized electrons in the covalent compounds, since they do not bond to any atoms and behave like "quasi" atoms in the lattice. ${ }^{4}$ The electronic structure reveals that these electrons occupied the interstitial electron bands near the Fermi level, making electrides as promising candidates to prepare conductive materials with unusual optical or magnetic properties, such as efficient thermionic emitters, ${ }^{5}$ low injection-barrier cathodes, ${ }^{6}$ and highperformance catalysts, ${ }^{7-10}$ etc.

Organic electrides are thermally and chemically unstable at room temperature and in air, limiting their practical application. ${ }^{2,3,11,12}$ Instead, inorganic electrides appear as the irreplaceable alternatives due to their improved thermal and chemical stability that is much more suitable for practical application. Till now, only a few inorganic electrides have been successfully synthesized thus far. The as-synthesized $\mathrm{C}_{12} \mathrm{~A}_{7}: \mathrm{e}^{-}\left(\left[\mathrm{Ca}_{24} \mathrm{Al}_{28} \mathrm{O}_{64}\right]\right.$ $\left.4^{+}\left(\mathrm{e}^{-}\right)_{4}\right)^{6}$ is a typical zero-dimensional (OD) electride with anionic electrons trapped in the center of one cage with 12 neighboring cages around it. The high-density, loosely bounded electrons with a small work function make it a promise for applications that require an efficient cold electron field-emitter competing with carbon nanotubes and diamond. The newly synthesized $\operatorname{Sr}_{5} \mathrm{P}_{31}{ }^{13}$ as a semiconductor with a distinct band gap, is an ideal one- dimensional (1D) electride. Recently, $\mathrm{Ca}_{2} \mathrm{~N}$ and $\mathrm{Y}_{2} \mathrm{C}$ are representative examples of two-dimensional (2D) electrides where anionic electrons are confined in the 2D interlayer space. ${ }^{14,15}$ Upon compression, $\mathrm{Ca}_{2} \mathrm{~N}$ experiences a phase transition from a $2 \mathrm{D}$ electride to a semiconducting $0 \mathrm{D}$ electride with the formation of anionic electrons localized in atom-sized cavities, ${ }^{16}$ which was later on confirmed by a subsequent experiment. ${ }^{17}$ For decades, $\mathrm{TiO}_{2}$ has been considered as a technically important material that is known to be an efficient photocatalyst for a wide range of applications (e.g., air and water purification, hydrogen evolution, and photoelectrochemical conversion, etc.). Other stoichiometries in $\mathrm{Ti}-\mathrm{O}$ materials, especially for O-rich $\mathrm{Ti}-\mathrm{O}$ materials (e.g., $\mathrm{TiO}$, $\mathrm{Ti}_{2} \mathrm{O}_{3}$, and $\mathrm{TiO}_{2}$ ) in different forms and types, also show the great potential as photocatalysts, together with other applications as ultrahard $^{18}$ and ultrastiff ${ }^{19}$ materials.

We note that Ti-rich $\mathrm{Ti}-\mathrm{O}$ materials are naturally electron-rich systems and thus they might have the potential to be the good candidate of electrides. This assumption seems to be in contradiction with a recent theoretical proposal that the formation probability of electride in the Ti-N system is rather low (about $3-4 \%)^{20}$ since Ti has a high electronegativity making it unfavorable for the electron donation to the lattice. For a deeper understanding of the nature of $\mathrm{Ti}$ element for the formation of electrides, we here have systematically explored the potential electrides in Ti-rich $\mathrm{Ti}-\mathrm{O}$ materials via first-principles calculations on electronic structures in conjunction with swarm structure searches. In this work, we predicted two new stoichiometries of $\mathrm{Ti}_{4} \mathrm{O}$ and $\mathrm{Ti}_{5} \mathrm{O}$ as good electrides that are likely to be synthesizable

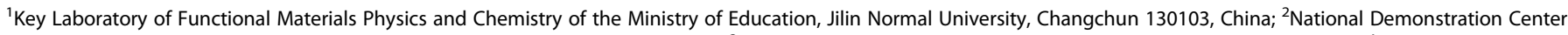
for Experimental Physics Education, Jilin Normal University, Siping 136000, China; ${ }^{3}$ Beijing Computational Science Research Center, Beijing 100193 , China; ${ }^{4}$ School of Physics and

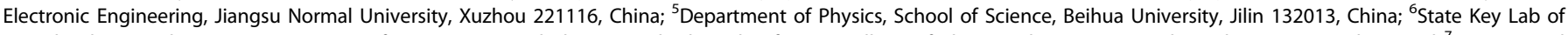

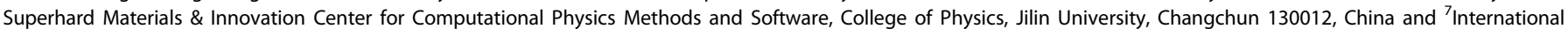
Center for Future Science, Jilin University, Changchun 130012, China

Correspondence: Hanyu Liu (Ihy@calypso.cn) or Yanming Ma (mym@calypso.cn)
}

Received: 20 August 2018 Accepted: 22 November 2018

Published online: 07 December 2018 
at $19 \mathrm{GPa}$ and ambient pressure. Our calculations reveal that the multiple cavities and layered atomic packings in the structure caused by " $\mathrm{Ti}_{6} \mathrm{O}$ " or " $\mathrm{Ti}_{7} \mathrm{O}$ " polyhedral units, making the excess electrons have an ideal habitat to stay in.

\section{RESULTS}

Applying the standard formal charges of $4+$ and $2-$ to the Ti and $\mathrm{O}$ atoms, respectively, the compounds $\mathrm{Ti}_{x} \mathrm{O}(x=2-6)$ would have at least 6 excess electrons per formula, indicating the intrinsic electron-rich feature. Our structure prediction begins on the three known phases of $\mathrm{Ti}_{2} \mathrm{O}, \mathrm{Ti}_{3} \mathrm{O}$, and $\mathrm{Ti}_{6} \mathrm{O}$ materials at ambient pressure, which are crystalized in $P-3 m 1, P-31 C$, and $P-31 C$ symmetry, respectively (the detailed structure information is shown in Table S1). To explore the possibility to form electrides in $\mathrm{Ti}_{2} \mathrm{O}, \mathrm{Ti}_{3} \mathrm{O}$, and $\mathrm{Ti}_{6} \mathrm{O}$ compounds, we have conducted a series of first-principles calculations on their electronic structures. The electronic band structure and the corresponding projected density of states (PDOS) are plotted in Fig. 1 and Figure S5, respectively. It is seen that all these three structures exhibit metallic feature. The conducting states mainly derive from Ti-3d states around the Fermi level. The $0-2 p$ states make a quite small contribution to the states near the Fermi level, but mainly occupy in the valance band. This result also demonstrates partial charge transfer from Ti-3d to the O-2p orbitals, which is further supported by the result of Bader charge calculation (Table 1). Obviously, excess electrons provided by Ti atoms are firstly being captured by $\mathrm{O}$ atoms with higher electronegativity. There, to reach the stable state of 8-electron closed shell configuration, each $\mathrm{O}$ atom can accept up to 2 more electrons. The rest of excess electrons might be trapped in the interstitial spaces, favorable for the formation of electrides. Subsequently, electron localization function (ELF) is carefully examined since ELF has been demonstrated as the efficient method to characterize the localization of excess electrons in the interstitial spaces unshared by any atomic orbitals. We calculated the ELF maps for $P-3 m 1$ phase of $\mathrm{Ti}_{2} \mathrm{O}, P-31 \mathrm{C}$ phase of $\mathrm{Ti}_{3} \mathrm{O}$, and $P-31 \mathrm{c}$ phase of $\mathrm{Ti}_{6} \mathrm{O}$ (Fig. 2 and Figure $\mathrm{S} 6$ ) at ambient pressure. Indeed, we found the formation of $\mathrm{OD}$ electrides since atomic-sized localized electrons are seen at the interstitial sites for these three structures with a typical choice of isosurface of 0.75 in ELF calculation, which often gives a common measure on the electron localization. ${ }^{20}$ Moreover, we have integrated the charge

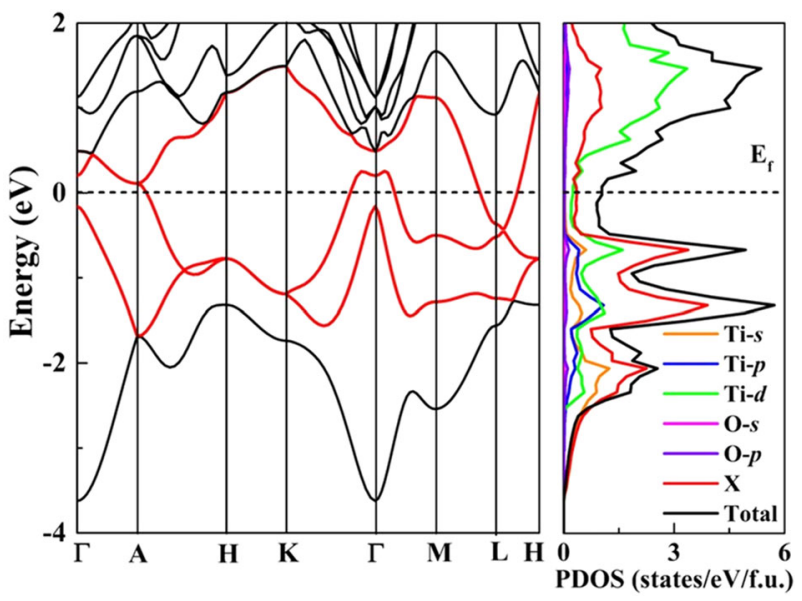

Fig. 1 Band structure (left panel) and projected density of states (PDOS) (right panel) of $P-3 m 1$ phase of $\mathrm{Ti}_{2} \mathrm{O}$ at ambient pressure. The dispersive band (red line) crossing the Fermi level $\left(E_{f}\right)$ represents the "interstitial band", which is mainly composed of the OD electrons confined in the interstitial regions as shown in Fig. $2 a$. The red line in the right panel denotes the contributions attributed to the virtual orbitals of the interstitial area. The black dashed lines in left and right panel indicate the Fermi level, marked as $E_{\mathrm{f}}$
Table 1. Residual charges on $\mathrm{Ti}$ atom, $\mathrm{O}$ atoms, and interstitial areas $(\mathrm{X})$ of phases in different stoichiometries based on the Bader charge analysis

\begin{tabular}{|c|c|c|c|c|}
\hline Phase & Pressure (GPa) & Ti (e/atom) & $\mathrm{O}$ (e/atom) & X (e/f.u.) \\
\hline $\mathrm{Ti}_{2} \mathrm{O}-\mathrm{P}-3 m 1$ & 0 & 19.05 & 9.78 & 4.12 \\
\hline $\mathrm{Ti}_{2} \mathrm{O}-\mathrm{Cmcm}$ & 50 & 20.64 & 9.71 & 1.01 \\
\hline $\mathrm{Ti}_{3} \mathrm{O}-\mathrm{Cmmm}$ & 0 & 20.68 & 9.77 & 2.19 \\
\hline $\mathrm{Ti}_{3} \mathrm{O}-$ Pmam & 50 & 21.2 & 9.75 & 0.65 \\
\hline $\mathrm{Ti}_{4} \mathrm{O}-\mathrm{Pmmm}$ & 50 & 21.25 & 9.77 & 1.23 \\
\hline $\mathrm{Ti}_{5} \mathrm{O}-\mathrm{P}-1$ & 0 & 20.74 & 9.83 & 4.4 \\
\hline $\mathrm{Ti}_{5} \mathrm{O}-\mathrm{C} 2 / \mathrm{m}$ & 50 & 21.64 & 9.78 & 0.0 \\
\hline $\mathrm{Ti}_{6} \mathrm{O}-P-31 \mathrm{C}$ & 0 & 20.73 & 9.85 & 5.77 \\
\hline $\mathrm{Ti}_{6} \mathrm{O}-\mathrm{Cmmm}$ & 50 & 21.33 & 9.77 & 2.25 \\
\hline
\end{tabular}

$\mathrm{X}$ denotes the charge transfer from $\mathrm{Ti}$ atom to interstitial area

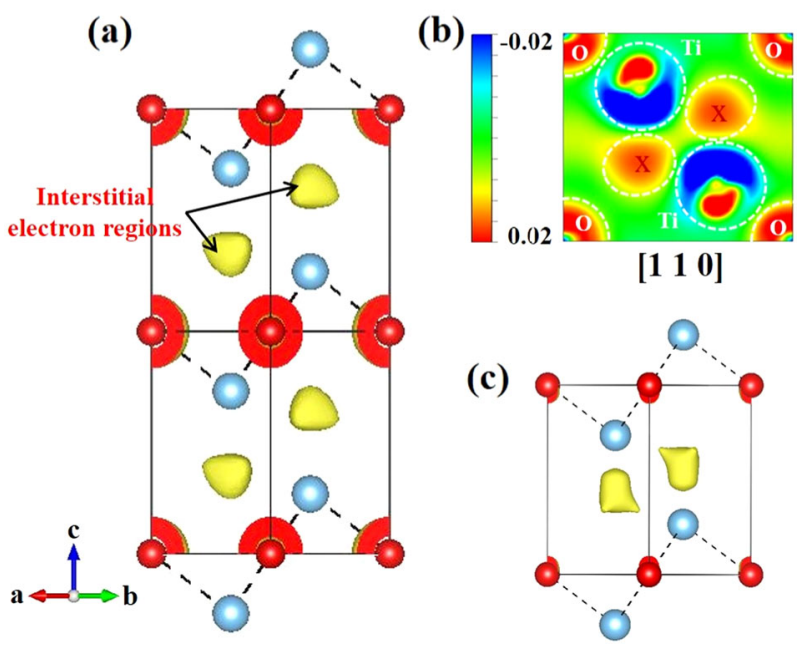

Fig. 2 For the $P-3 m 1$ phase of $\mathrm{Ti}_{2} \mathrm{O}$ : a Calculated electron localization functions (ELFs) with an isosurface of 0.75 . The atomsized localized electrons in the interstitial regions are marked in yellow. b Charge density difference on the $(110)_{R}$ plane. The positions of $\mathrm{Ti}$ and $\mathrm{O}$ atoms and the localized electrons in the interstitial regions are marked as " $\mathrm{Ti}$ ", " $\mathrm{O}$ ", and " $\mathrm{X}$ ", respectively. The charge difference was calculated relative to the superposition of atomic charges. c Partial charge density around the Fermi level $-1.7 \mathrm{eV}<E-E_{\mathrm{f}}<0 \mathrm{eV}$

of the localized region. If the integrated charge is larger than one, this compound is likely to be considered as an electride.

\section{DISCUSSION}

In these intrinsic electron-rich systems, we take the $P-3 m 1$ phase of $\mathrm{Ti}_{2} \mathrm{O}$ as a typical example. Subsequent calculations have been performed to characterize the electronic properties and will be discussed below. According to the calculated partial charge densities around the Fermi level (Fig. 2c), the bands crossing the Fermi level in left panel of Fig. 1 correspond to electrons confined within the interstitial sites. There is a strong ionic interaction between the cationic $\mathrm{Ti}$ atom and anionic localized electrons, making the interstitial bands quite dispersive. This situation is similar to those in previously reported electrides, such as $\mathrm{CaN}_{2}{ }^{15,16} \mathrm{Y}_{2} \mathrm{C}_{1}^{14}$ etc. ${ }^{13,43}$ In order to identify the contributions from the interstitial electrons and avoid it being projected onto the $3 d$ orbitals of the neighboring $\mathrm{Ti}$ atoms, we placed a Wigner-Seitz radius of $1.42 \AA$ in the lattice of the $P$ - $3 m 1$ phase 
and obtained the PDOS curves for the interstitial site as shown in right panel of Fig. 1. We note that the contribution from the interstitial sites is stronger than the PDOS for $\mathrm{Ti}$ and $\mathrm{O}$ atoms near Fermi surface. The interstitial bands show a strong hybridization between Ti-3d and Ti-4s orbitals, indicating that the $s-d$ hybridization plays a crucial role in the formation of strong nonnuclear charge maxima from the expulsion of valence electrons into the interstitial regions of the lattice.

For a better understanding of the electronic band structures, it is necessary to trace these interstitial electrons. In Fig. 2a, we plotted out the ELF map, which clearly shows the formation of anionic localized electrons in the interstitial cavities of the lattice, representing the formation of a $0 \mathrm{D}$ electride. The charge density difference on the $(110)_{R}$ plane also clearly indicate adequate electrons transfer from $\mathrm{Ti}$ atoms to the interstitial areas, see Fig. $2 \mathrm{~b}$. Furthermore, the existence of these interstitial states is further confirmed by the partial charge densities as seen in Fig. 2c. We have integrated the electrons of interstitial regions in PDOS (Fig. 2) and found that it is $\sim 3 \mathrm{e}^{-}$per cavity.

In addition to the known ambient pressure structures of $\mathrm{Ti}_{2} \mathrm{O}$, $\mathrm{Ti}_{3} \mathrm{O}$, and $\mathrm{Ti}_{6} \mathrm{O}$ discussed above, we below explore the so far undiscovered stoichiometric compounds and new phases which also have the possibility of being potential electrides. The structure prediction calculations on the Ti-rich stoichiometries of $\mathrm{Ti}_{x} \mathrm{O}(x=1-6)$ with the cell sizes of 1-6 formula units (f.u.) are performed by using the CALYPSO method. We systematically evaluated the predicted structures via their formation enthalpies relative to the products of dissociation into constituent elements (i.e., solidified phases of $\mathrm{Ti}$ and $\mathrm{O}_{2}$ ) up to $100 \mathrm{GPa}$ at $0 \mathrm{~K}$.

$\Delta H\left(\mathrm{Ti}_{x} \mathrm{O}\right)=\left[h\left(\mathrm{Ti}_{x} \mathrm{O}\right)-x h(\mathrm{Ti})-h(\mathrm{O})\right] /(x+1)$

where $\Delta H$ and $h$ values represent formation enthalpies and enthalpies, respectively. We then construct the convex hull at each pressure, as depicted in Fig. 3a. All the structures whose formation enthalpies lie on the convex hull (solid lines) are deemed to be thermodynamically stable with respect to decomposition into elements or other binary compounds, and thus, are experimentally synthesizable in principle. At ambient pressure, our structure searching calculations successfully reproduced the experimentally synthesized $P-3 m 1$ phase of $\mathrm{Ti}_{2} \mathrm{O}, P-31 \mathrm{C}$ phase of $\mathrm{Ti}_{3} \mathrm{O}$, and $P-31 \mathrm{C}$ phase of $\mathrm{Ti}_{6} \mathrm{O} .{ }^{44}$ Meanwhile, a new stoichiometry $\mathrm{Ti}_{5} \mathrm{O}$ structure (Fig. 4f) appears on the convex hull being thermodynamically stable at ambient pressure, to the best of our knowledge, which has not reported in the previous literature. For $\mathrm{Ti}_{3} \mathrm{O}$, we have discovered an energetically more favorable structure $(\mathrm{Cmmm}$ phase, see Fig. 4c) marked as red solid triangle on the convex hull, with a $\Delta H$ value of $0.08 \mathrm{eV} /$ atom lower than $P-31 C$ phase (the red circle above the convex hull). The two structures are rather different as it can be seen in the simulated distinct $X$-ray diffraction patterns (Figure S3). Both the phases are dynamically stable as seen in their phonon spectra (Figure S4). At $50 \mathrm{GPa}$, it is clearly seen that a new stoichiometry $\mathrm{Ti}_{4} \mathrm{O}$ (Fig. 4e) emerges on the convex hull. The stable pressure ranges of the corresponding structures were calculated and are shown in Fig. $3 \mathrm{~b}$.

The predicted stable structures are shown in Fig. 4a-i (see Supplementary Table S1 for detailed structural information). For $\mathrm{Ti}_{2} \mathrm{O}$, the ambient pressure trigonal $P-3 m 1$ phase transforms to the orthorhombic $\mathrm{Cmcm}$ structure at $26 \mathrm{GPa}$. Both the structures clearly show staggered layered packing pattern. The averaged $\mathrm{Ti}-\mathrm{O}$ bond length of $2.15 \AA$ is reduced to $2.04 \AA$. As to $\mathrm{Ti}_{3} \mathrm{O}$, the orthorhombic $\mathrm{Cmmm}$ phase transforms to orthorhombic Pmam phase at $10 \mathrm{GPa}$, where the edge-linked $" \mathrm{Ti}_{6} \mathrm{O}$ " changes into distorted atom-linked octahedron unit, with the Ti-O bond length being reduced from 2.11 to $2.00 \AA$. The $\mathrm{Ti}_{4} \mathrm{O}$ can be stabilized above $\sim 19 \mathrm{GPa}$, exhibiting an orthorhombic Pmmm structure with isolated $\mathrm{Ti}$ atoms at each vertex position. At $0 \mathrm{GPa}$, the predicted stable phase of $\mathrm{Ti}_{5} \mathrm{O}$ adopts a triclinic $P-1$ structure. Upon compression to $39 \mathrm{GPa}$, it transforms to a monoclinic $\mathrm{C} 2 / \mathrm{m}$

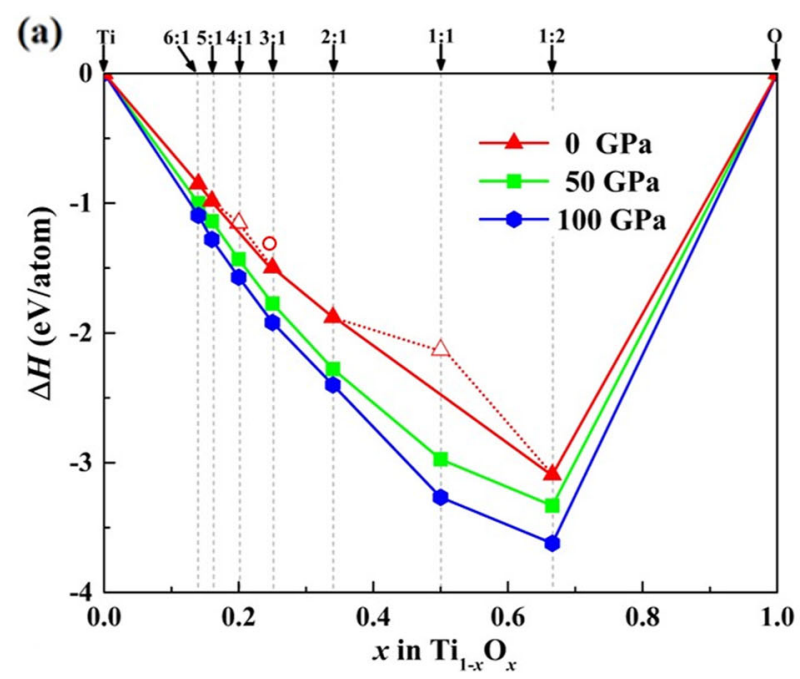

(b)

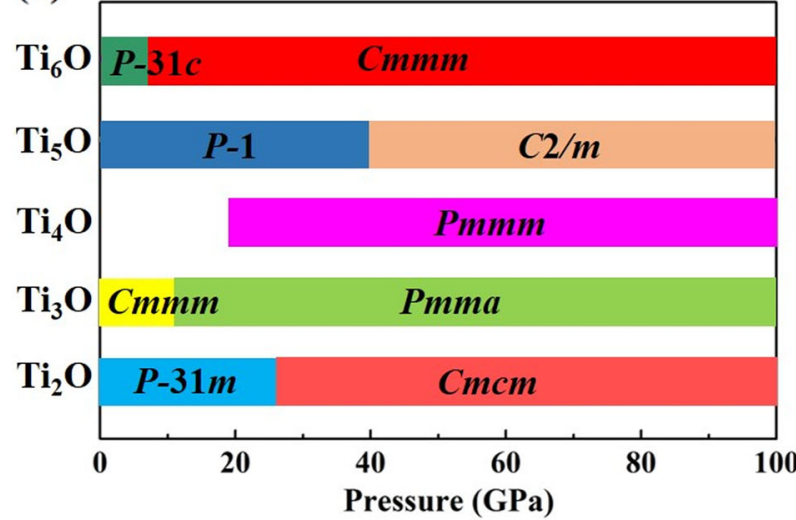

Fig. 3 a Formation enthalpies $(\Delta H)$ of various Ti-rich Ti-oxides with respect to decomposition into constituent elemental solids at 0-100 GPa. Data points located on the convex hull (solid lines) represent stable species against any type of decomposition. We also added the experimental $P-31 c$ structure into the convex hull (the red circle). $\mathbf{b}$ Pressure ranges in which the corresponding structures of different stoichiometries are stabilized. The $a$-phase $\left(\mathrm{PG}_{3} / \mathrm{mmc}\right)$ and $\omega$-phase $(P 6 / \mathrm{mmm})$ of $\mathrm{Ti}$, and the $\zeta$-phase $(\mathrm{C2} / \mathrm{m})$ of $\mathrm{O}_{2}$ are used for calculating $\Delta H$

structure with the formation of " $\mathrm{Ti}_{7} \mathrm{O}$ " decahedron unit of each $\mathrm{O}$ atom coordinated by $7 \mathrm{Ti}$ atoms, the highest coordination number of $\mathrm{O}$ atom in the Ti-rich Ti-O system. As to the Ti-richest stoichiometry $\mathrm{Ti}_{6} \mathrm{O}$, the known experimental trigonal $P-31 \mathrm{C}$ structure transforms to an orthorhombic $\mathrm{Cmmm}$ structure, both of which share the " $\mathrm{Ti}_{6} \mathrm{O}$ " octahedron unit with some isolated $\mathrm{Ti}$ atoms sitting in the interstitial sites. All the newly predicted structures have the layered or cage-like open structures composed of the $" \mathrm{Ti}_{6} \mathrm{O}$ " or " $\mathrm{Ti}_{7} \mathrm{O}$ " polyhedral units, which can readily accommodate excess electrons in the interstitial regions. Our phonon calculations have verified that the newly predicted structures are dynamically stable by the evidence of the absence of any imaginary frequency in the whole Brillouin zone (Figure S4). In addition, we have also calculated the phonon dispersion of $\mathrm{Ti}_{4} \mathrm{O}$ at $0 \mathrm{GPa}$ [Figure S4(e)]. There is also no imaginary frequency in the whole Brillouin zone, indicating $\mathrm{Ti}_{4} \mathrm{O}$ in the $\mathrm{Pmmm}$ structure is likely to be recovered as a metastable phase at ambient pressure.

The calculated ELF maps of the newly predicted structures indicate the electrides in nature of these Ti-rich oxides by exhibiting atomic-sized electrons localized in the interstitial areas (Figure S6). The electronic band structures and the PDOS results reveal the metallic feature of these structures (Figure S5). Due to 
(a)

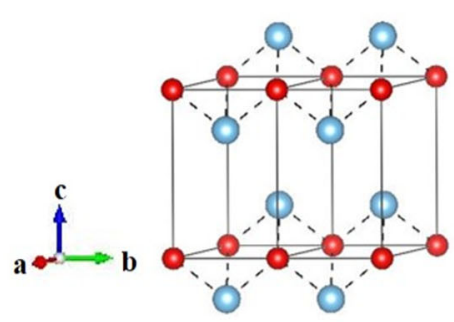

(d)

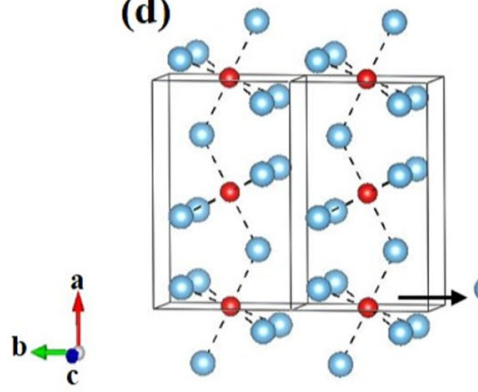

(g)

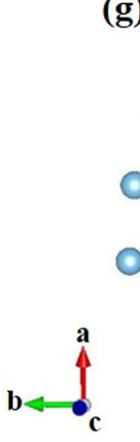

(b)

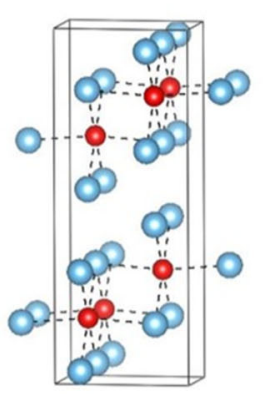

(e)

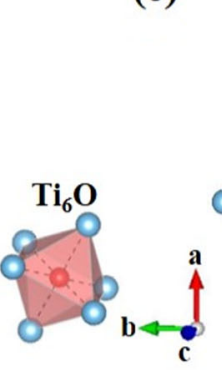

(h)

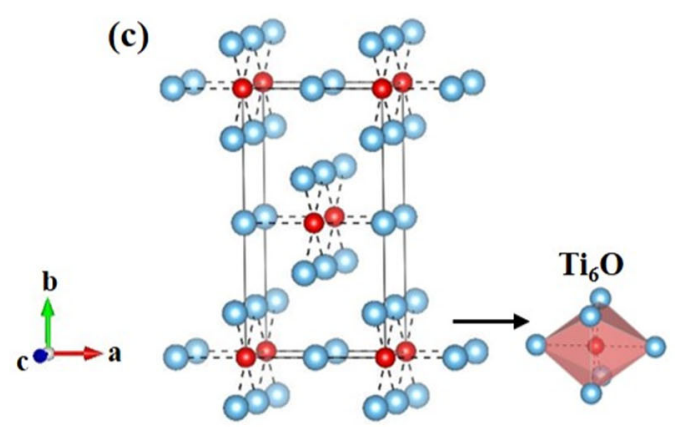

(f)
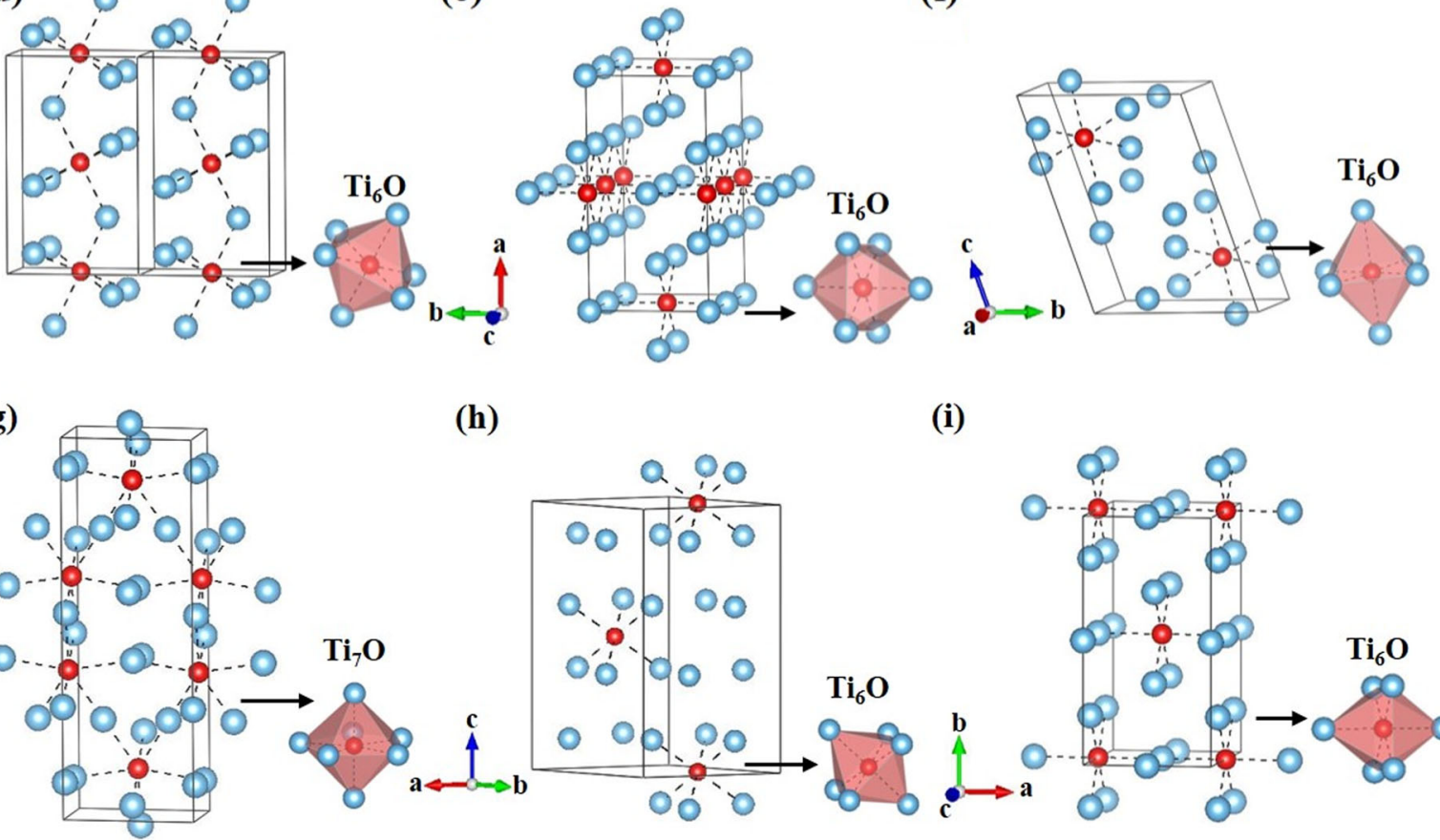

(i)
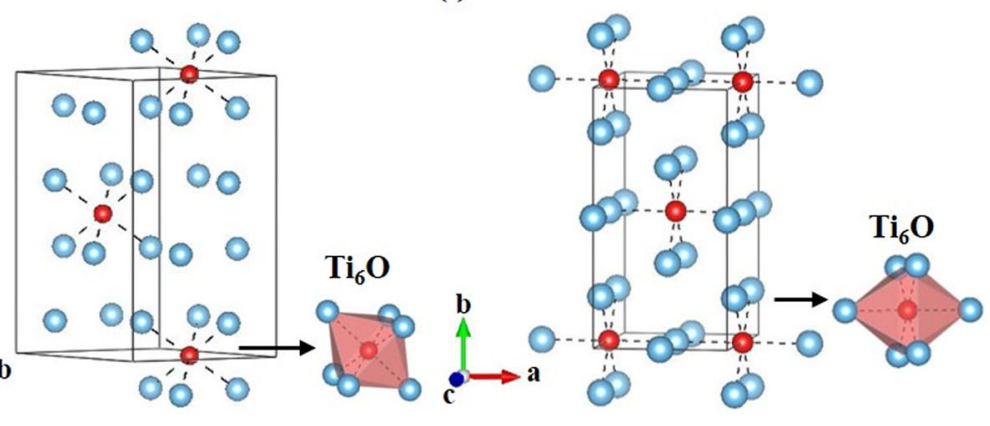

Fig. 4 Structures of stable Ti-rich Ti-oxides at high pressures: $\mathbf{a} \mathrm{Ti}_{2} \mathrm{O}$ in the $\mathrm{P}-3 m 1$ structure; $\mathbf{b} \mathrm{Ti}_{2} \mathrm{O}$ in the $\mathrm{Cmcm}$ structure; $\mathbf{c ~ T i}{ }_{3} \mathrm{O}$ in the $\mathrm{Cmmm}$ structure; $\mathbf{d} \mathrm{Ti}_{3} \mathrm{O}$ in the $P$ mam structure; $\mathbf{e} \mathrm{Ti}_{4} \mathrm{O}$ in the $P m m m$ structure; $\mathbf{f} \mathrm{Ti}_{5} \mathrm{O}$ in the $P-1$ structure; $\mathbf{g} \mathrm{Ti}_{5} \mathrm{O}$ in the $C 2 / m$ structure; $\mathbf{h} \mathrm{Ti}_{6} \mathrm{O}$ in the $P-$ $31 \mathrm{c}$ structure; $\mathbf{i ~ T i}_{6} \mathrm{O}$ in the $\mathrm{Cmmm}$ structure. The small red and large blue spheres represent $\mathrm{O}$ and $\mathrm{Ti}$ atoms, respectively. Solid lines depict the unit cells of the structures. The dash line between Ti and $\mathrm{O}$ atom denotes the ionic bonding pattern. See the Supplemental Material for more detailed structural information

the fact that spherical charge integration around the ELF basins is confined by the actual area and shape of interstitial electrons, the integration from Bader charge basins may underestimate the total interstitial charge. The electron counts with total and transferred charges are shown for each ion in different stoichiometries (Table 1).

We found that electron-rich condition for a compound is necessary but not sufficient in forming electride. Structure patterns able to provide open interstitial regions play also an irreplaceable role in the stabilization of electrides. Though most of our newly predicted Ti-rich oxides are good electrides, the ambient pressure $a$-phase, high pressure $\mathrm{NaCl}$-type, ${ }^{45,46}$ and $\mathrm{CsCl}$-type ${ }^{34}$ phases of Ti-rich $\mathrm{TiO}$ compound are not candidates of electrides since the structures are rather compact without exhibiting any atom-sized cavity in the lattice. Even for our predicted $\mathrm{Ti}_{5} \mathrm{O}$ compound, once the electride structure at ambient pressure transforms into the high-pressure phase of $\mathrm{C} 2 / \mathrm{m}$ structure (Fig. 4g), electron localization in the lattice is destroyed with the loss of electride in nature.

In summary, we predicted through first-principles calculations on electronic structures of Ti-rich oxides that three known ambient pressure structures of Ti-rich $\mathrm{Ti}_{2} \mathrm{O}, \mathrm{Ti}_{3} \mathrm{O}$, and $\mathrm{Ti}_{6} \mathrm{O}$ compounds are electrides. Our swarm-intelligence structure searches identified two new stoichiometries of $\mathrm{Ti}_{4} \mathrm{O}$ and $\mathrm{Ti}_{5} \mathrm{O}$ that have not been reported before in the literature, which are considered as electrides and likely to be synthesizable above a pressure of $19 \mathrm{GPa}$. The formation of electrides in these structures is attributed to the presence of peculiar structure feature of " $\mathrm{Ti}_{6} \mathrm{O}$ " or " $\mathrm{Ti}_{7} \mathrm{O}$ " polyhedral units that give rise to the atom-sized cavities or the interlayer spacial regions in the lattice to accommodate excess electrons for the stabilization of electrides. Our current findings represent a step forward to the comprehensive understanding of electrides in the technically important Ti-O system, particularly for the Ti-rich oxides.

\section{METHODS}

For structure relaxations, we choose the Perdew-Burke-Ernzerhof generalized gradient approximation ${ }^{21}$ as implemented in the VASP code. ${ }^{22}$ After a throughout test, we choose the kinetic cutoff energy of $500 \mathrm{eV}$ and Monkhorst-Pack ${ }^{23}$ Brillouin zone sampling grid with a resolution of $2 \pi \times$ $0.03 \AA^{-1}$ to ensure that enthalpy could converge with several meV per atom. In some cases, the total energy can converge as $1 \mathrm{meV} /$ atom (Figure S2). The all-electron projector-augmented wave (PAW) ${ }^{24}$ method was adopted, where $3 d^{2} 4 s^{2}$ and $2 s^{2} 2 p^{4}$ are treated as valence electrons for $\mathrm{Ti}$ 
and $\mathrm{O}$ atoms, respectively. To test the validity of the selected PAW potentials for $\mathrm{Ti}$ and $\mathrm{O}$ in application to high pressure conditions, we have compared the equation of states (EOS) of the $\mathrm{Cmcm}$ structure of $\mathrm{Ti}_{2} \mathrm{O}$ using both PAW method adopted in this work and the full potential method. ${ }^{21,25}$ Identical EOS data by using both methods are obtained (see Figure S1) to validate the PAW pseudopotentials adopted up to $100 \mathrm{GPa}$. To assess the dynamical stability of the various Ti-rich Ti-O compounds, the harmonic approximation was used to calculate their phonon spectra within the supercell method. ${ }^{26,27}$

Our structural prediction approach is based on a global minimization of free energy surfaces of given compounds by combining ab initio totalenergy calculations with the particle swarm optimization (PSO) algorithm. ${ }^{21}$ The structure search of each $\mathrm{Ti}_{x} \mathrm{O}(x=1-6)$ stoichiometry is performed with simulation cells containing 1-4 formula units. In the first generation, a population of structures belonging to certain space group symmetries are randomly constructed. Local optimizations of candidate structures are done by using the conjugate gradients method through the VASP code, ${ }^{22}$ with an economy set of input parameters and an energy convergence threshold of $1 \times 10^{-5} \mathrm{eV}$ per cell. Starting from the second generation, $60 \%$ structures in the previous generation with the lower enthalpies are selected to produce the structures of next generation by the PSO operators. The $40 \%$ structures in the new generation are randomly generated. A structure fingerprinting technique of bond characterization matrix is employed to evaluate each newly generated structure, and identical structures are strictly forbidden. These procedures significantly enhance the diversity of sampled structures during the evolution, which is crucial in driving the search into the global minimum. For most of the cases, the structure search for each chemical composition converges (evidenced by no structure with the lower energy emerging) after 1000-1200 structures investigated (i.e., in about 20-30 generations).

The ELF was calculated to determine the bonding nature and the excess electrons trapped in the interstitial sites. Bader charge calculations ${ }^{28}$ have been performed to quantitatively analyze the charge transfer situation. To find the "missing" stable $\mathrm{Ti}_{x} \mathrm{O}$ structures, we performed the structure searching simulations via the cutting-edge CALYPSO method, ${ }^{29-32}$ which enables global minimization of energy surfaces by merging ab initio totalenergy calculations. The effectiveness of this method is benchmarked on various known systems. ${ }^{14,18,33-42}$ The electronic structures of the most stable structures were calculated using the same settings as the structure re-relaxation step, except denser Monkhorst-Pack grids with a spacing of around $2 \pi \times 0.01 \AA^{-1}$ was used in the density of states (DOS) calculations.

\section{DATA AVAILABILITY}

All data generated or analyzed during this study are included in this published article (and its supplementary information files).

\section{ACKNOWLEDGEMENTS}

The authors acknowledge funding from the National Natural Science Foundation of China under Grant Nos. 11704151, 11774127, U1530124, 61475063, 61505067, 11504007, and 11534003; the Science Challenge Project, No. TZ2016001; Science Challenge Project, No. TZ2016001; Program for JLU Science and Technology Innovative Research Team (JLUSTIRT); the program for JLU Science and Technology Innovative Research Team, No. 201705; The Scientific and Technological Research Project of the "13th Five-Year Plan" of Jilin Provincial Education Department under Grant Nos. JJKH20180772KJ, JJKH20180769KJ, JJKH20180761KJ, and 201648. We utilized computing facilities at the High Performance Computing Center of Jilin University and Tianhe2-JK at the Beijing Computational Science Research Center.

\section{AUTHOR CONTRIBUTIONS}

X.Z. and Y.M. conceived the research; X.Z. performed the simulations and analyzed the data with the help of M.X., Lili Yang, X.Q., Lihua Yang, and M.Z.; X.Z. wrote the manuscript; H.L. and Y.M helped to revise the manuscript. All authors discussed and commented on the manuscript.

\section{ADDITIONAL INFORMATION}

Supplementary information accompanies the paper on the npj Computational Materials website (https://doi.org/10.1038/s41524-018-0131-6).

Competing interests: The authors declare no competing interests.
Publisher's note: Springer Nature remains neutral with regard to jurisdictional claims in published maps and institutional affiliations.

\section{REFERENCES}

1. Dye, J. L. Electrides: early examples of quantum confinement. Acc. Chem. Res. 42, 1564-1572 (2009).

2. Singh, D. J., Krakauer, H., Haas, C. \& Pickett, W. E. Theoretical determination that electrons act as anions in the electride Cs+ (15-crown-5)2·e-. Nature 365, 39-42 (1993).

3. Dye, J. L. Electrides: ionic salts with electrons as the anions. Science 247, 663-668 (1990).

4. Miao, M. \& Hoffmann, R. High-pressure electrides: the chemical nature of interstitial quasiatoms. J. Am. Chem. Soc. 137, 3631-3637 (2015).

5. Toda, Y. et al. Intense thermal field electron emission from room-temperature stable electride. Appl. Phys. Lett. 87, 254103 (2005).

6. Matsuishi, S. High-density electron anions in a nanoporous single crystal: $\left[\mathrm{Ca}_{24} \mathrm{Al}_{28} \mathrm{O}_{64}\right]_{4}+(4 \mathrm{e}-)$. Science 301, 626-629 (2003).

7. Kitano, M. et al. Ammonia synthesis using a stable electride as an electron donor and reversible hydrogen store. Nat. Chem. 4, 934-940 (2012).

8. Toda, Y. et al. Activation and splitting of carbon dioxide on the surface of an inorganic electride material. Nat. Commun. 4, 2378 (2013).

9. Kitano, M. et al. Electride support boosts nitrogen dissociation over ruthenium catalyst and shifts the bottleneck in ammonia synthesis. Nat. Commun. 6, 6731 (2015).

10. Sharif, M. J. et al. Electron donation enhanced $\mathrm{CO}$ oxidation over Ru-loaded $12 \mathrm{CaO} \cdot 7 \mathrm{Al}_{2} \mathrm{O}_{3}$ electride catalyst. J. Phys. Chem. C 119, 11725-11731 (2015).

11. Wagner, M. J. \& Dye, J. Alkalides, electrides, and expanded metals. Annu. Mater. Sci. 23, 223-253 (1993).

12. Ellaboudy, A., Dye, J. L. \& Smith, P. B. Cesium 18-crown-6 compounds. A crystalline ceside and a crystalline electride. J. Am. Chem. Soc. 105, 6490-6491 (1983).

13. Wang, J. et al. Exploration of stable strontium phosphide-based electrides: theoretical structure prediction and experimental validation. J. Am. Chem. Soc. 139, 15668-15680 (2017).

14. Zhang, $X$. et al. Two-dimensional transition-metal electride $Y_{2} C$. Chem. Mater. 26, 6638-6643 (2014).

15. Lee, K., Kim, S. W., Toda, Y., Matsuishi, S. \& Hosono, H. Dicalcium nitride as a twodimensional electride with an anionic electron layer. Nature 494, 336-340 (2013).

16. Zhang, Y., Wu, W., Wang, Y., Yang, S. A. \& Ma, Y. Pressure-stabilized semiconducting electrides in alkaline-Earth-metal subnitrides. J. Am. Chem. Soc. 139, 13798-13803 (2017).

17. Tang, H. et al. Metal-to-semiconductor transition and electronic dimensionality reduction of $\mathrm{Ca}_{2} \mathrm{~N}$ electride under pressure. Adv. Sci. 5, 1800666 (2018).

18. Zhang, M. et al. Crystal structures of $\mathrm{CaB}_{3} \mathrm{~N}_{3}$ at high pressures. Inorg. Chem. 56, 7449-7453 (2017).

19. Dubrovinskaia, N. A. et al. Experimental and theoretical identification of a new high-pressure $\mathrm{TiO}_{2}$ polymorph. Phys. Rev. Lett. 87, 275501 (2001).

20. Zhang, Y., Wang, H., Wang, Y., Zhang, L. \& Ma, Y. Computer-assisted inverse design of inorganic electrides. Phys. Rev. X 7, 011017 (2017).

21. Perdew, J. P., Burke, K. \& Ernzerhof, M. Generalized gradient approximation made simple. Phys. Rev. Lett. 77, 3865-3868 (1996).

22. Kresse, G. \& Furthmüller, J. Efficient iterative schemes for ab initio total-energy calculations using a plane-wave basis set. Phys. Rev. B 54, 11169-11186 (1996).

23. Pack, J. D. \& Monkhorst, H. J. 'Special points for Brillouin-zone integrations'-a reply. Phys. Rev. B 16, 1748-1749 (1977).

24. Kresse, G. \& Joubert, D. From ultrasoft pseudopotentials to the projector augmented-wave method. Phys. Rev. B 59, 1758-1775 (1999).

25. The Elk FP-LAPW Code. http://elk.sourceforge.net/.

26. Giannozzi, P., de Gironcoli, S., Pavone, P. \& Baroni, S. Ab initio calculation of phonon dispersions in semiconductors. Phys. Rev. B 43, 7231-7242 (1991).

27. Baroni, S., Giannozzi, P. \& Testa, A. Green's-function approach to linear response in solids. Phys. Rev. Lett. 58, 1861-1864 (1987).

28. Bader, R. F. W., Popelier, P. L. A. \& Keith, T. A. Theoretical definition of a functional group and the molecular orbital paradigm. Angew. Chem. Int. Ed. Engl. 33, 620-631 (1994).

29. Wang, Y., Lv, J., Zhu, L. \& Ma, Y. Crystal structure prediction via particle-swarm optimization. Phys. Rev. B 82, 094116 (2010).

30. Wang, Y. et al. An effective structure prediction method for layered materials based on 2D particle swarm optimization algorithm. J. Chem. Phys. 137, 224108 (2012).

31. Wang, Y., Lv, J., Zhu, L. \& Ma, Y. CALYPSO: a method for crystal structure prediction. Comput. Phys. Commun. 183, 2063-2070 (2012).

32. Lv, J., Wang, Y., Zhu, L. \& Ma, Y. Particle-swarm structure prediction on clusters. J. Chem. Phys. 137, 084104 (2012). 
33. Zhong, X. et al. Tellurium hydrides at high pressures: high-temperature superconductors. Phys. Rev. Lett. 116, 057002 (2016).

34. Zhong, X. et al. Crystal structures and electronic properties of oxygen-rich titanium oxides at high pressure. Inorg. Chem. 57, 3254-3260 (2018).

35. Miao, M. et al. Anionic chemistry of noble gases: formation of Mg-NG (NG $=\mathrm{Xe}$, $\mathrm{Kr}, \mathrm{Ar}$ ) compounds under pressure. J. Am. Chem. Soc. 137, 14122-14128 (2015).

36. Zhang, W. et al. Unexpected stable stoichiometries of sodium chlorides. Science 342, 1502-1505 (2013).

37. Wang, H., Tse, J. S., Tanaka, K., litaka, T. \& Ma, Y. Superconductive sodalite-like clathrate calcium hydride at high pressures. Proc. Natl. Acad. Sci. U.S.A. 109, 6463-6466 (2012).

38. Peng, F., Miao, M., Wang, H., Li, Q. \& Ma, Y. Predicted lithium-boron compounds under high pressure. J. Am. Chem. Soc. 134, 18599-18605 (2012).

39. Liu, H., Naumov, I. I., Hoffmann, R., Ashcroft, N. W. \& Hemley, R. J. Potential high- $T_{\mathrm{c}}$ superconducting lanthanum and yttrium hydrides at high pressure. Proc. Natl. Acad. Sci. U.S.A. 114, 6990-6995 (2017).

40. Zhong, $X$. et al. Pressure stabilization of long-missing bare $C_{6}$ hexagonal rings in binary sesquicarbides. Chem. Sci. 5, 3936-3940 (2014).

41. Zhang, S., Wang, Y., Yang, G. \& Ma, Y. Silicon framework-based lithium silicides at high pressures. ACS Appl. Mater. Interfaces 8, 16761-16767 (2016).

42. Zhu, L., Liu, H., Pickard, C. J., Zou, G. \& Ma, Y. Reactions of xenon with iron and nickel are predicted in the Earth's inner core. Nat. Chem. 6, 644-648 (2014).

43. Ming, W., Yoon, M., Du, M.-H., Lee, K. \& Kim, S. W. First-principles prediction of thermodynamically stable two-dimensional electrides. J. Am. Chem. Soc. 138, 15336-15344 (2016).
44. Kornilov, I. I. et al. Neutron diffraction investigation of ordered structures in the titanium-oxygen system. Metall. Trans. 1, 2569 (1970)

45. Valeeva, A. A., Rempel', A. A. \& Gusev, A. I. Ordering of cubic titanium monoxide into monoclinic $\mathrm{Ti}_{5} \mathrm{O}_{5}$. Inorg. Mater. 37, 603-612 (2001).

46. Bartkowski, S. et al. Electronic structure of titanium monoxide. Phys. Rev. B 56, 10656-10667 (1997)

(C) Open Access This article is licensed under a Creative Commons Attribution 4.0 International License, which permits use, sharing, adaptation, distribution and reproduction in any medium or format, as long as you give appropriate credit to the original author(s) and the source, provide a link to the Creative Commons license, and indicate if changes were made. The images or other third party material in this article are included in the article's Creative Commons license, unless indicated otherwise in a credit line to the material. If material is not included in the article's Creative Commons license and your intended use is not permitted by statutory regulation or exceeds the permitted use, you will need to obtain permission directly from the copyright holder. To view a copy of this license, visit http://creativecommons. org/licenses/by/4.0/.

c The Author(s) 2018 